\title{
CRISPR/Cas-mediated chromosome engineering: opening up a new avenue for plant breeding
}

\author{
Michelle Rönspies ,Patrick Schindele and Holger Puchta*, \\ Botanical Institute, Karlsruhe Institute of Technology, Fritz-Haber-Weg 4, 76133 Karlsruhe, Germany \\ * Correspondence: holger.puchta@kit.edu
}

Editor: John Lunn, MPI of Molecular Plant Physiology, Germany

\begin{abstract}
The advent of powerful site-specific nucleases, particularly the clustered regularly interspaced short palindromic repeats (CRISPR)/CRISPR-associated protein (Cas) system, which enables precise genome manipulation, has revolutionized plant breeding. Until recently, the main focus of researchers has been to simply knock-in or knock-out single genes, or to induce single base changes, but constant improvements of this technology have enabled more ambitious applications that aim to improve plant productivity or other desirable traits. One long-standing aim has been the induction of targeted chromosomal rearrangements (crossovers, inversions, or translocations). The feasibility of this technique has the potential to transform plant breeding, because natural rearrangements, like inversions, for example, typically present obstacles to the breeding process. In this way, genetic linkages between traits could be altered to combine or separate favorable and deleterious genes, respectively. In this review, we discuss recent breakthroughs in the field of chromosome engineering in plants and their potential applications in the field of plant breeding. In the future, these approaches might be applicable in shaping plant chromosomes in a directed manner, based on plant breeding needs.
\end{abstract}

Keywords: Arabidopsis, chromosomal rearrangements, CRISPR/Cas9, gene editing, genome engineering, inversion, plant breeding, translocation.

\section{Introduction}

By crossing and selecting plants with desirable characteristics, humankind has already been genetically manipulating crop plants for thousands of years. Following advances in molecular biology and high-throughput genotyping, breeding efficiency has steadily improved. However, further improvement of current so-called elite crops is limited, e.g. due to the occurrence of linkage-drag or reduced genetic diversity caused by the domestication process (Wolter et al., 2019). The emergence of a new approach, referred to as genome editing, has enabled the precise manipulation of genes by site-specific nucleases such as the clustered regularly interspaced short palindromic repeats (CRISPR)/CRISPR associated protein (Cas) system (Jinek et al., 2012). Its simple composition of a Cas protein and a single guide RNA, together with its ease of use has made the CRISPR/Cas system the method of choice for various applications in plant genome editing (Chen et al., 2019; Schindele 
et al., 2020). The induction of double-strand breaks (DSBs) by the Cas nuclease is the crucial step, which stimulates errorprone non-homologous end-joining (NHEJ) or homologydirected repair mechanisms (Puchta, 2005). NHEJ is the predominant repair mechanism in somatic plant cells. Here, re-ligation of the broken ends occasionally results in mutations such as smaller insertions or deletions at the break site (Puchta and Fauser, 2014). If two DSBs are induced simultaneously, this pathway can be harnessed to accomplish large-scale chromosomal rearrangements. These can cover chromosomal inversions, translocations, or deletions (Schmidt et al., 2020b). Since its introduction as a genome editing tool in 2012 (Jinek et al., 2012), the CRISPR/Cas system has been constantly improved. Whereas, so far, researchers have mainly focused on the knock-in or knock-out of single genes or the induction of single base changes, the development of more powerful genome editing tools has shifted the focus to previously unprecedented approaches. This Expert View aims to cover recent breakthroughs in the field of CRISPR/Cas-mediated chromosome engineering in plants (Box 1). They represent the first steps towards being truly able to shape chromosomes in a directed manner (e.g. by breaking or establishing genetic linkages in crop plants) and they offer new possibilities, e.g. in imitating genome evolution.

\section{Transferring new traits into crop plants}

Current elite crops have been selected for desirable traits, such as high yield or improved flavor. However, by selecting those traits, others, such as resistance against several stress conditions

\section{Box 1. Key developments in establishing CRISPR/Cas-mediated genome engineering in plants}

\section{- De-novo domestication accelerates plant breeding}

Wild relatives of cultured crop plants offer genetic diversity, which has been lost in many crops during the domestication process. Li et al. (2018) and Zsögön et al. (2018) have demonstrated that CRISPR/ Cas-mediated multiplex editing of 'domestication genes' (e.g. loci associated with desirable traits) can dramatically accelerate the domestication process of a tomato ancestor, Solanum pimpinellifolium. Subsequently, Lemmon et al. (2018) identified and edited orthologues of tomato domestication genes in another member of the Solanaceae family, Physalis pruinosa, and succeeded in improving the plants' growth habit and fruit size within one generation.

\section{- Targeted crossovers can be achieved by inducing somatic homologous recombination}

Filler Hayut et al. (2017) demonstrated that CRISPR/Cas-induced targeted double-strand breaks (DSBs) can be repaired by somatic homologous recombination (HR) using a homologous chromosome as a template. They were able to identify gene conversions and a putative crossover event that could, unfortunately, not be transferred to the next generation. This study demonstrates that, in principle, 'targeted crossovers' via DSB-induced somatic HR can be accomplished.

\section{- First successful induction of heritable inversions in the Mb range in Arabidopsis}

It was shown for the first time by Schmidt et al. (2019) that heritable inversions of up to $18 \mathrm{~kb}$ can be induced in Arabidopsis, using the Cas9 orthologue from Staphylococcus aureus, under the control of an egg cell-specific promoter. In a follow-up study, Schmidt et al. (2020a) succeeded in reversing the wellknown 1.17 Mb hk4S inversion, a heterochromatic knob, in Arabidopsis accession Columbia, using the same CRRISPR/Cas tool as in their previous study. It was demonstrated that meiotic recombination could be restored in the formerly crossover-inaccessible inversion between homologous chromosomes of a Columbia-line harboring the reversed knob ('rknob') and a knob-less accession, Landsberg erecta.

\section{- First CRISPR/Cas-mediated reciprocal translocations achieved in Arabidopsis}

Beying et al. (2020) recently reported the first CRISPR/Cas-induced reciprocal chromosomal translocations in Arabidopsis. The authors were able to establish a method for generating heritable targeted translocations. Reciprocal translocations were induced between chromosome pairs 1 and 2 , and chromosomes 1 and 5 , with a size of about $1 \mathrm{Mb}$ and $0.5 \mathrm{Mb}$, respectively. 
or nutritional value, were lost (Gruber, 2017). Further improvement of these elite crops by traditional breeding techniques is limited due to the minimal genetic diversity between different cultivars, which has been caused by the long selective domestication process. Introgression of new traits also strongly depends on the occurrence of crossovers (COs) during meiotic recombination to generate novel allelic combinations However, the number of COs is typically limited to one to three per chromosome, and COs accumulate prevalently in so-called 'recombination hot spots' (Mercier et al., 2015). Therefore, adjacent genes from recombination-poor regions are often inherited together. If this so-called linkage drag involves undesired genetic material, many rounds of backcrossing are required to remove the linkage by classical breeding techniques (Hasan et al., 2015). Typically, genetic linkages can only be disrupted by naturally occurring meiotic recombination events (Taagen et al., 2020). However, the constant development of more efficient CRISPR/Cas tools has introduced new possibilities in this area. Multiplexing provides a novel approach to the induction of chromosomal rearrangements, the generation of genetic diversity, and the manipulation of meiotic recombination. Recent breakthroughs in these areas will be discussed in the following section.

\section{Creation of genetic diversity via de novo domestication}

Uncultured varieties of commercial crop plants offer 'unlocked' genetic potential, but the transfer of desirable traits into elite crops by classical breeding can take many years (Southgate et al., 1995). However, by targeting multiple, so-called domestication genes (e.g. involved in plant architecture, fruit size, or fruit number) with genome editing tools, this process can be drastically accelerated. Three recent studies demonstrated that de novo domestication can be accomplished using CRISPR/ Cas9. The first two studies were published in the same issue of Nature Biotechnology and described the de novo domestication process in a tomato ancestor, Solanum pimpinellifolium, using CRISPR/Cas9 (Zsögön et al., 2018; Li et al., 2018). The third study edited domestication genes in a tomato relative, Physalis pruinosa, despite the previous lack of reference genomes and efficient transformation protocols (Lemmon et al., 2018).

In the first study, Zsögön et al. (2018) simultaneously edited six loci involved in yield and productivity in the wild tomato ancestor, S. pimpinellifolium, to combine favorable traits from commercial and wild tomatoes. Loss-of-function alleles were created using a multiplexing CRISPR/Cas9 approach. The targets included SELF-PRUNING (SP; growth habit), OVATE (fruit shape), FASCIATED (fruit size), FRUIT WEIGHT (fruit weight), MULTIFLORA (fruit number), and LYCOPENE BETA CYCLASE (nutritional value). Only edited alleles were recovered in the $\mathrm{T}_{1}$ generation, demonstrating the high editing efficiency of this approach. Compared with wild $S$. pimpinellifolium, fruit size was increased 3-fold and fruit numbers were increased 10-fold. Also, the nutritional quality was improved due to an increase of the lycopene content by $500 \%$. All these changes were achieved within a single generation.

In the second study, Li et al. (2018) targeted four stress tolerant wild tomato accessions of $S$. pimpinellifolium (resistant against bacterial spot disease or salt stress). They used a multiplex CRISPR/Cas9 editing approach to target coding sequences, as well as cis-regulatory elements and upstream open reading frames, of genes involved in shoot architecture $(S P)$, flowering time (SELF PRUNING 5G; SP5G), and fruit size (CLAVATA3 and WUSCHEL). In contrast to Zsögön et al., who recovered only edited alleles, Li et al. observed a variety of mutations, with one to four genes being edited. The plants with all four genes edited showed earlier flowering, determinate growth, and increased fruit size, while retaining their original stress resistance.

In the third study, Lemmon et al. (2018) subjected another member of the Solanaceae family, P. pruinosa, to de novo domestication. Due to their lack of reference genomes, orthologues of tomato domestication genes were identified and selected for editing. To improve the plants' weedy growth habit and small fruit size, three targets were edited, including the $P$. pruinosa equivalents of SP and SP5G (growth habit), as well as CLAVATA1 (CLV1; fruit size). Plants with edited SP showed a more compact growth habit, but limited fruit production was also observed. As a target that resulted in fewer negative effects on fruit production upon editing, SP5G was edited. In this case, the authors again observed a more compact growth, but also a higher fruit number. In an attempt to increase fruit size, CLV1 was targeted to increase locule numbers. Editing of this target resulted in a $24 \%$ increase in fruit mass.

\section{Chromosome engineering: finally possible}

So far, the CRISPR/Cas tools have mainly been used to target single or multiple genes, e.g. by editing open reading frames, regulatory regions, or altering genome and epigenome structure through Cas-mediated scaffolds (Schindele et al., 2020). The recent improvement and development of more powerful genome editing tools has shifted the focus to more ambitious approaches, such as the manipulation of meiotic recombination or large-scale chromosomal restructuring. These may pave the way for novel synthetic plant biology applications. For example, in 2018, CRISPR/Cas-mediated chromosome engineering was successfully used for the generation of a singlechromosome and two-chromosome yeast (Shao et al. (2018) and Luo et al. (2018), respectively).

An obvious possibility for applying targeted chromosomal rearrangements in plant breeding is the induction of crossovers between homologous chromosomes, which aims to manipulate meiotic recombination in certain genomic regions. This approach concentrates on the control of $\mathrm{CO}$ rates and $\mathrm{CO}$ distribution. During meiosis, homologous recombination 
(HR) serves to pair homologous chromosomes for subsequent COs until they become separated again (Lambing et al., 2017). This step also plays an essential role in plant breeding, because meiotic recombination allows for novel allelic combinations. Therefore, manipulation of the number and position of COs has been a longstanding goal. In a novel approach, meiotic recombination was induced at naturally low-recombination sites in the yeast Saccharomyces cerevisiae by tethering the Spo11 protein, a subunit of the meiosis initiation machinery, to sitespecific DNA-binding modules (Sarno et al., 2017). These included zinc finger nucleases, transcription activator-like effector nucleases and catalytically inactive Cas9 scaffolds. All approaches were able to stimulate $\mathrm{CO}$ frequencies in naturally recombination-cold regions, although with only modest efficiency. Some regions were still inaccessible for targeted DSB induction, which might indicate a putative limitation of relying on proteins of the natural meiosis machinery for DSB induction. In contrast to other eukaryotes, plant germ cells differentiate at a late developmental stage (Wang and $\mathrm{Ma}$, 2011), which allows for the inheritance of somatically obtained mutations. Therefore, DSBs do not have to be induced exclusively during meiosis to manipulate genome structures. In an outstanding study, Filler Hayut et al. (2017) demonstrated that targeted DSBs can induce somatic HR using a homologous chromosome as template. In this study, a selection system was developed in tomato hybrids to identify HR between homologous chromosomes, based on a visual marker gene (PSY1) and single-nucleotide polymorphisms (SNPs). Through induction of allele-specific DSBs, using CRISPR/Cas9, Filler Hayut et al. were able to identify somatic HR events, including gene conversions and a putative crossover event that, unfortunately, could not be transferred to the next generation. This study demonstrated that 'targeted COs' via DSB-induced somatic HR can be accomplished. This could provide another way to break genetic linkages but, more importantly, it could
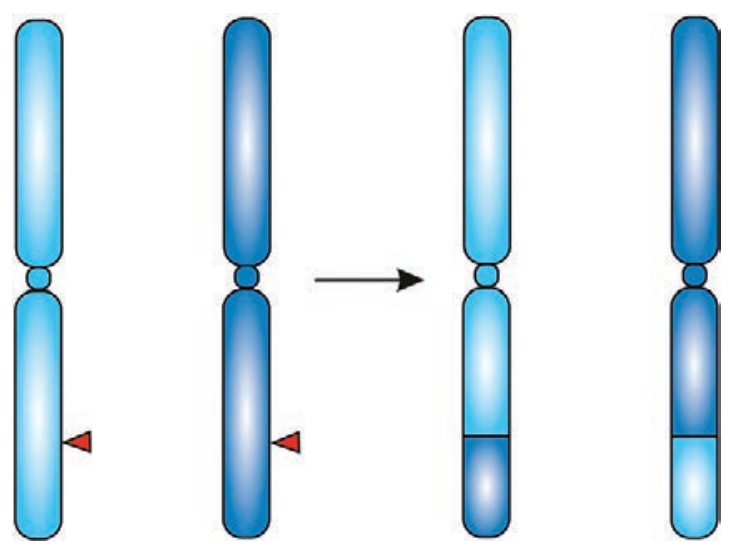

Fig. 1. Manipulation of meiotic recombination by generation of targeted COs. If CRISPR/Cas-induced DSBs on both homologous chromosomes are repaired by somatic or meiotic $\mathrm{HR}$, with the respective homologous chromosome as a template, targeted COs can be generated. Red triangles indicate CRISPR/Cas-mediated DSBs. also make genetic recombination attainable in a controllable manner (Fig. 1).

Additionally, the CRISPR/Cas system can be utilized to induce chromosomal restructuring. Controlling those kinds of rearrangements can be considered as the next level in plant breeding, because it will help overcome previous bottlenecks. The simultaneous induction of two DSBs can result in inversions or deletions, if they are induced on the same chromosome. They can also lead to translocations, if the DSBs are induced on two different chromosomes. So far, these rearrangements have mainly been induced on a small scale, e.g. by inverting single genes. CRISPR/Cas-mediated inversions of up to $18 \mathrm{~kb}$ have been reported in Arabidopsis, by utilizing the Staphylococcus aureus Cas9 nuclease in combination with an egg cell-specific promoter (Schmidt et al., 2019). Editing efficiencies of up to $10 \%$ were reached in single $\mathrm{T}_{2}$ lines. In contrast, targeted CRISPR/Cas-induced translocations have not been reported until recently.

Natural chromosomal inversions often span several Mbs. If they occur in a heterozygous state, they might lead to unbalanced gametes and reduced fertility following crossover events during meiosis (Fransz et al., 2016). Nevertheless, inversions are abundant in natural populations, which could be linked to their association with genome evolution, adaptation, or speciation (Kirkpatrick and Barton, 2006; Fang et al., 2012). In heterozygotes, genes located in inverted regions are not accessible for genetic reshuffling through COs, as genetic exchange is suppressed between homologous chromosomes within the inversion (Wellenreuther and Bernatchez, 2018). One of the best-known inversions in Arabidopsis is the paracentric hk4S inversion on chromosome 4 , a heterochromatic knob, which has a size of $1.17 \mathrm{Mb}$ and is carried by around 170 accessions, one of them being Columbia (Fransz et al., 2000, 2016). It has been shown that in crosses between the knob-carrying accession Columbia and a knob-less accession, Landsberg erecta, genetic exchange between the inverted sequences cannot be detected (Drouaud et al., 2006). Recently, in a follow-up study to the obtained $18 \mathrm{~kb}$ inversion (Schmidt et al., 2020a), the heterochromatic knob in Arabidopsis Columbia was successfully reversed, using the same combination of $S$. aureus Cas 9 with an egg cell-specific promoter, which had proven to be efficient in generating heritable inversions in the previous study (Schmidt et al. 2019). This demonstrated for the first time that, using the CRISPR/Cas system, inversions in the Mb range could be induced and stably inherited in plants. The inversion was induced via two CRISPR/Cas9-mediated DSBs close to the previously identified borders of the knob (Fransz et al., 2016), which led to the inversion of the complete $1.17 \mathrm{Mb}$ knob fragment. The study further aimed to investigate whether meiotic recombination within the formerly CO-inaccessible knob could be restored in hybrids harboring the reverted knob in a heterozygous state. Therefore, crosses between Columbia plants harboring the reverted knob ('rknob') and the knobless accession, Landsberg erecta, were conducted. Using a 
A
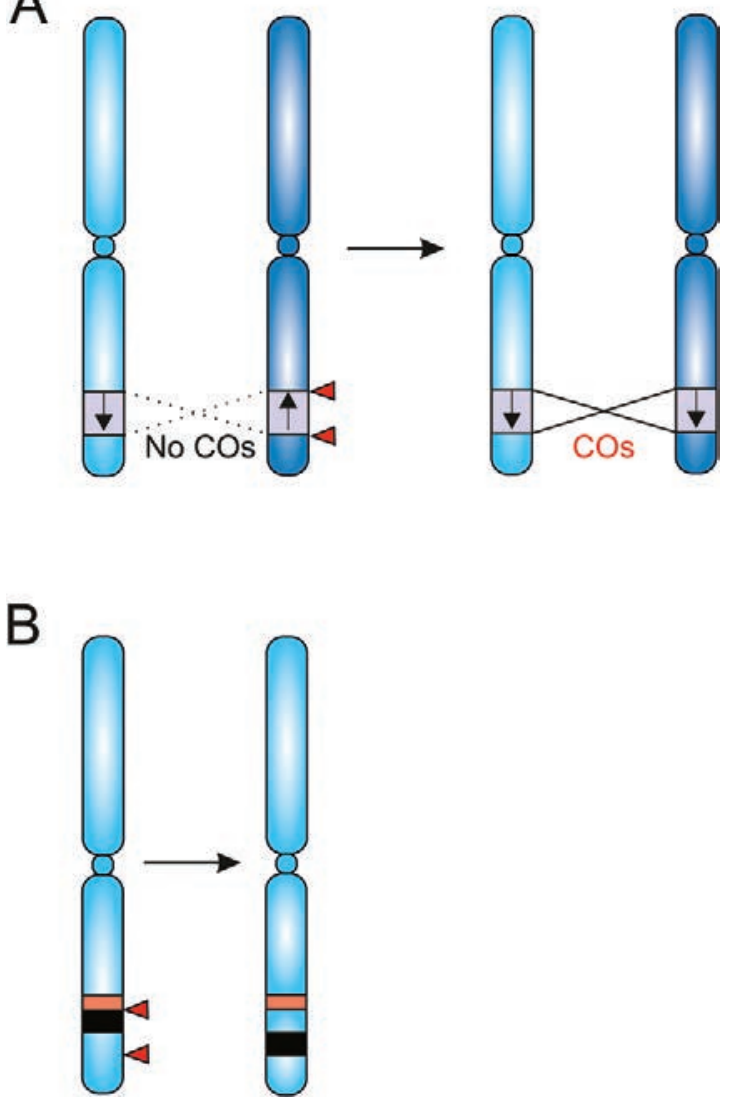

Fig. 2. CRISPR/Cas-induced inversions can restore $\mathrm{CO}$ rates in formerly inverted regions and can break genetic linkage groups. (A) Quantitative trait loci located in evolutionarily derived inversions are inaccessible to meiotic recombination and, therefore, plant breeding. They can be made accessible for $\mathrm{COs}$ again by reversing the inversion through two induced DSBs at the borders of the inversion. Red triangles indicate CRISPR/Casmediated DSBs. (B) Targeted inversions can serve to break genetic linkage groups. In this case, one DSB needs to be induced between two linked genes, resulting in the physical separation of the two genes upon inversion induction. Red triangles indicate CRISPR/Cas-mediated DSBs.

SNP-based recombination assay, it was revealed that CO rates could be restored within the previously inverted fragment. This demonstrates that evolutionarily emerged inversions can be reversed and that the restoration of meiotic recombination in a previously inaccessible region using an efficient CRISPR/Cas system is attainable. This technique could be applied in plant breeding to make quantitative trait loci, located in evolutionarily derived inversions, accessible to meiotic recombination again by reverting the inversion (Fig. 2A). Moreover, the targeted induction of inversions could be useful in breaking genetic linkages, by choosing one Cas9 DSB site between the two linked genes, which results in the physical separation of the two genes (Fig. 2B).

In contrast to CRISPR/Cas-induced inversions, there had not been any reports about CRISPR/Cas-mediated translocations in plants prior to this year. They had only been observed as a by-product of CRISPR/Cas genome editing
A
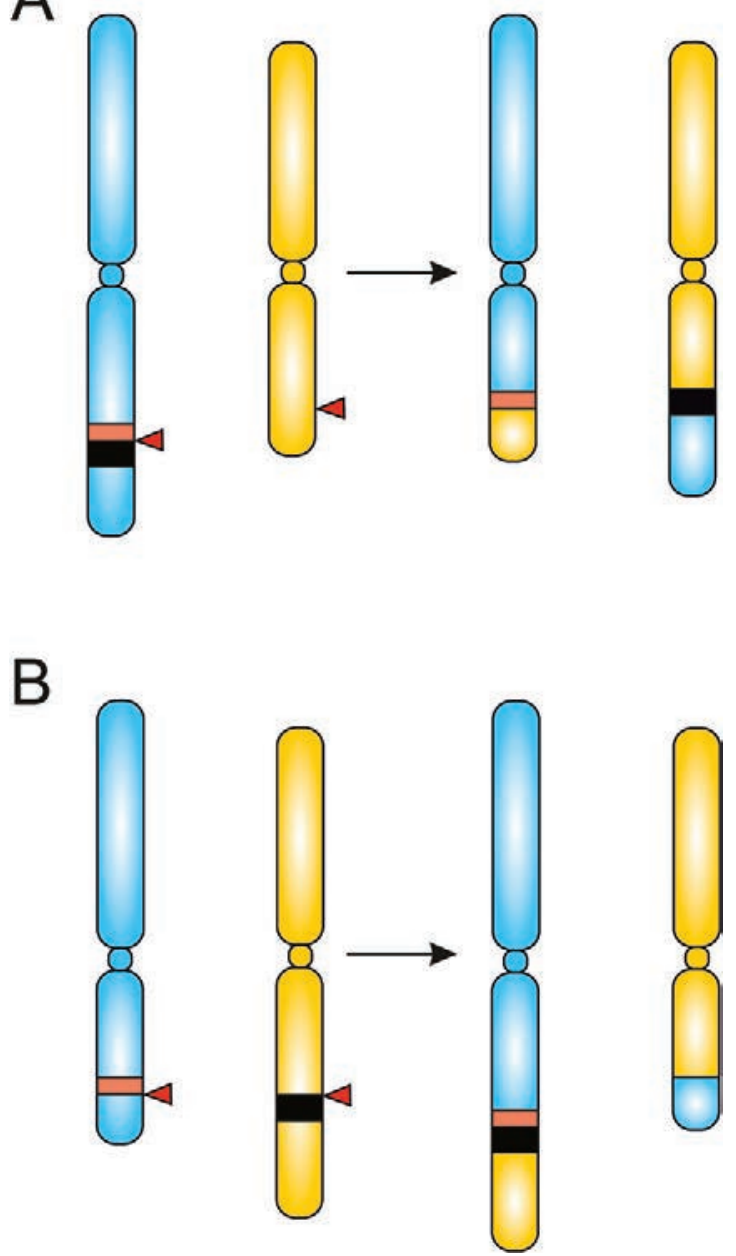

Fig. 3. CRISPR/Cas-induced translocations can break or generate genetic linkage groups. (A) If one DSB is induced between two linked genes, and the other DSB is induced on a different chromosome, reciprocal translocations can lead to the physical separation of genes in a linkage group. Red triangles indicate CRISPR/Cas-mediated DSBs. (B) If two genes need to be propagated together, genetic linkage groups can be created by inducing a translocation to move the desired genes in close proximity to each other. If the two DSBs are respectively induced above and beneath the desired genes, reciprocal translocations can lead to the creation of a new linkage group. Red triangles indicate CRISPR/Casmediated DSBs.

(Peterson et al., 2016). In a groundbreaking study, CRISPR/ Cas-induced reciprocal chromosomal translocations were recently achieved in Arabidopsis (Beying et al., 2020). The authors were able to establish a method of generating heritable targeted translocations. Reciprocal translocations were induced between chromosome 1 and 2, and chromosome 1 and 5 , with a size of about $1 \mathrm{Mb}$ and $0.5 \mathrm{Mb}$, respectively. The translocation frequencies were found to be up to $2.5 \%$ in the wild type background and up to $3.75 \%$ in the classical NHEJ mutant ku70. Like inversions, translocations play a role in speciation and genome evolution (Lysak et al., 2006; Gabur et al., 2019). The targeted induction of translocations 
provides another opportunity for the breakage, or the fixation, of genetic linkages (Fig. 3). In this way, not only can deleterious genes be decoupled, but desirable traits can also be permanently linked to assure common propagation.

\section{Conclusion}

Steady advancements of CRISPR/Cas genome editing tools now enable not only the targeted modification of genes, but also the rearrangement of large chromosomal fragments. As demonstrated by recent accomplishments, such as the induction of chromosomal inversions and translocations, this technique is finally applicable for chromosome engineering approaches in plants. These proof-of-concept studies have shown that the targeted induction of inversions and translocations can, in principle, be used to break genetic linkages with deleterious genes, or to generate linkages between favorable genes, even genes from different chromosomes. These approaches could eventually provide access to a new realm of synthetic biology. Apart from the removal of inhibitory DNA sequences, targeted chromosomal rearrangements pave the way for the creation of synthetic plant chromosomes. As chromosomal rearrangements are often associated with genome evolution and speciation, e.g. by leading to reproductive isolation, even the creation of new species might become feasible by mimicking the processes of genome evolution. However, the use of efficient multiplex editing tools is crucial for these applications (Zhang et al., 2019). In addition, before these new techniques can be applied in crop plants, some hurdles, such as low transformation efficiencies and more demanding genome structures, must still be overcome.

\section{Acknowledgements}

The authors wish to thank Daniel Donahey for proofreading the manuscript. This work was supported by the European Research Council (Advanced Grant 'CRISBREED').

\section{References}

Beying N, Schmidt C, Pacher M, Houben A, Puchta H. 2020. CRISPRCas9-mediated induction of heritable chromosomal translocations in Arabidopsis. Nature Plants 6, 638-645.

Chen K, Wang Y, Zhang R, Zhang H, Gao C. 2019. CRISPR/Cas genome editing and precision plant breeding in agriculture. Annual Review of Plant Biology 70, 667-697.

Drouaud J, Camilleri C, Bourguignon PY, et al. 2006. Variation in crossing-over rates across chromosome 4 of Arabidopsis thaliana reveals the presence of meiotic recombination "hot spots". Genome research 16, 106-114.

Fang Z, Pyhäjärvi T, Weber AL, et al. 2012. Megabase-scale inversion polymorphism in the wild ancestor of maize. Genetics 191, 883.

Filler Hayut S, Melamed Bessudo C, Levy AA. 2017. Targeted recombination between homologous chromosomes for precise breeding in tomato. Nature Communications 8, 15605.
Fransz P, Linc G, Lee CR, et al. 2016. Molecular, genetic and evolutionary analysis of a paracentric inversion in Arabidopsis thaliana. The Plant Journal 88, 159-178.

Fransz PF, Armstrong S, de Jong JH, Parnell LD, van Drunen C, Dean C, Zabel P, Bisseling T, Jones GH. 2000. Integrated cytogenetic map of chromosome arm 4S of $A$. thaliana: structural organization of heterochromatic knob and centromere region. Cell 100, 367-376.

Gabur I, Chawla HS, Snowdon RJ, Parkin IAP. 2019. Connecting genome structural variation with complex traits in crop plants. Theoretical and Applied Genetics 132, 733-750.

Gruber K. 2017. Agrobiodiversity: the living library. Nature 544, S8-S10.

Hasan MM, Rafii MY, Ismail MR, Mahmood M, Rahim HA, Alam MA, Ashkani S, Malek MA, Latif MA. 2015. Marker-assisted backcrossing: a useful method for rice improvement. Biotechnology \& Biotechnological Equipment 29, 237-254

Jinek M, Chylinski K, Fonfara I, Hauer M, Doudna JA, Charpentier E. 2012. A programmable dual-RNA-guided DNA endonuclease in adaptive bacterial immunity. Science 337, 816.

Kirkpatrick M, Barton N. 2006. Chromosome inversions, local adaptation and speciation. Genetics 173, 419-434.

Lambing C, Franklin FC, Wang CR. 2017. Understanding and manipulating meiotic recombination in plants. Plant physiology 173, 1530-1542.

Lemmon ZH, Reem NT, Dalrymple J, Soyk S, Swartwood KE, Rodriguez-Leal D, Van Eck J, Lippman ZB. 2018. Rapid improvement of domestication traits in an orphan crop by genome editing. Nature Plants 4, 766-770.

Li T, Yang X, Yu Y, et al. 2018. Domestication of wild tomato is accelerated by genome editing. Nature Biotechnology 36, 1160-1163.

Luo J, Sun X, Cormack BP, Boeke JD. 2018. Karyotype engineering by chromosome fusion leads to reproductive isolation in yeast. Nature $\mathbf{5 6 0}$ 392-396.

Lysak MA, Berr A, Pecinka A, Schmidt R, McBreen K, Schubert I. 2006. Mechanisms of chromosome number reduction in Arabidopsis thaliana and related Brassicaceae species. Proceedings of the National Academy of Sciences, USA 103, 5224-5229.

Mercier R, Mézard C, Jenczewski E, Macaisne N, Grelon M. 2015 The molecular biology of meiosis in plants. Annual Review of Plant Biology 66, 297-327

Peterson BA, Haak DC, Nishimura MT, Teixeira PJ, James SR, Dangl JL, Nimchuk ZL. 2016. Genome-wide assessment of efficiency and specificity in CRISPR/Cas9 mediated multiple site targeting in Arabidopsis. PLoS One 11, e0162169.

Puchta H. 2005. The repair of double-strand breaks in plants: mechanisms and consequences for genome evolution. Journal of Experimental Botany 56, 1-14. Puchta H, Fauser F. 2014. Synthetic nucleases for genome engineering in plants: prospects for a bright future. The Plant Journal 78, 727-741.

Sarno R, Vicq Y, Uematsu N, Luka M, Lapierre C, Carroll D, Bastianelli G, Serero A, Nicolas A. 2017. Programming sites of meiotic crossovers using Spo11 fusion proteins. Nucleic Acids Research 45, e164.

Schindele A, Dorn A, Puchta H. 2020. CRISPR/Cas brings plant biology and breeding into the fast lane. Current Opinion in Biotechnology 61, 7-14.

Schmidt C, Fransz P, Rönspies M, Dreissig S, Fuchs J, Heckmann S, Houben A, Puchta H. 2020a. Changing local recombination patterns in Arabidopsis by CRISPR/Cas mediated chromosome engineering. Nature Communications 11, 4418.

Schmidt C, Pacher M, Puchta H. 2019. Efficient induction of heritable inversions in plant genomes using the CRISPR/Cas system. The Plant Journal 98, 577-589.

Schmidt C, Schindele P, Puchta H. 2020b. From gene editing to genome engineering: restructuring plant chromosomes via CRISPR/Cas. aBIOTECH 1, 21-31.

Shao Y, Lu N, Wu Z, et al. 2018. Creating a functional single-chromosome yeast. Nature 560, 331-335. 
Southgate EM, Davey MR, Power JB, Marchant R. 1995. Factors affecting the genetic engineering of plants by microprojectile bombardment Biotechnology Advances 13, 631-651.

Taagen E, Bogdanove AJ, Sorrells ME. 2020. Counting on crossovers: controlled recombination for plant breeding. Trends in Plant Science 25, 455-465.

Wang Y, Ma H. 2011. Development: a pathway to plant female germ cells. Current Biology 21, R476-R478.

Wellenreuther M, Bernatchez L. 2018. Eco-evolutionary genomics of chromosomal inversions. Trends in Ecology \& Evolution 33, 427-440.
Wolter F, Schindele P, Puchta H. 2019. Plant breeding at the speed of light: the power of CRISPR/Cas to generate directed genetic diversity at multiple sites. BMC Plant Biology 19, 176.

Zhang Y, Malzahn AA, Sretenovic S, Qi Y. 2019. The emerging and uncultivated potential of CRISPR technology in plant science. Nature Plants 5, 778-794.

Zsögön A, Čermák T, Naves ER, et al. 2018. De novo domestication of wild tomato using genome editing. Nature Biotechnology 36, 1211-1216. 
Karlsruher Institut für Technologie

\section{Repository KITopen}

Dies ist ein Postprint/begutachtetes Manuskript.

Empfohlene Zitierung:

Rönspies, M.; Schindele, P.; Puchta, H.

CRISPR/Cas-mediated chromosome engineering: opening up a new avenue for plant breeding.

2021. (J. Lunn, Hrsg.)The journal of experimental botany, 72.

doi: $\underline{10.5445 / / R / 1000135477}$

Zitierung der Originalveröffentlichung:

Rönspies, M.; Schindele, P.; Puchta, H.

CRISPR/Cas-mediated chromosome engineering: opening up a new avenue for plant breeding.

2021. (J. Lunn, Hrsg.)The journal of experimental botany, 72 (2), 177-183.

doi: $10.1093 / j x b / e r a a 463$ 